New windows on massive stars: asteroseismology, interferometry, and spectropolarimetry

Proceedings IAU Symposium No. 307, 2014

G. Meynet, C. Georgy, J. H. Groh 8 Ph. Stee, eds.

(C) International Astronomical Union 2015 doi:10.1017/S1743921314007108

\title{
The magnetic field of $\zeta$ Ori A
}

\section{A. Blazère ${ }^{1}$, C. Neiner ${ }^{1}$, J-C. Bouret ${ }^{2}$, A. Tkachenko ${ }^{3} \dagger$ and the MiMeS collaboration}

${ }^{1}$ LESIA, Observatoire de Paris, UMR 8109 du CNRS, UPMC, Université Paris-Diderot, 5 place Jules Janssen, 92195 Meudon, France email: aurore.blazere@obspm.fr

${ }^{2}$ Aix-Marseille University, CNRS, LAM, UMR 7326, 13388 Marseille, France

${ }^{3}$ Instituut voor Sterrenkunde, KU Leuven, Celestijnenlaan 200D, B-3001 Leuven, Belgium

\begin{abstract}
Magnetic fields play a significant role in the evolution of massive stars. About $7 \%$ of massive stars are found to be magnetic at a level detectable with current instrumentation (Wade et al. 2013) and only a few magnetic O stars are known. Detecting magnetic field in O stars is particularly challenging because they only have few, often broad, lines to measure the field, which leads to a deficit in the knowledge of the basic magnetic properties of O stars. We present new spectropolarimetric Narval observations of $\zeta$ Ori A. We also provide a new analysis of both the new and older data taking binarity into account. The aim of this study was to confirm the presence of a magnetic field in $\zeta$ Ori A. We identify that it belongs to $\zeta$ Ori Aa and characterize it.
\end{abstract}

Keywords. stars: magnetic fields, stars: early-type, stars: individual ( $\zeta$ Ori A)

\section{Introduction}

A magnetic field seems to have been detected in the supergiant O 9.5I star $\zeta$ Ori A (Bouret et al. 2008). This magnetic field is the weakest ever reported in a massive star. Thanks to this measurement of the magnetic field one can locate $\zeta$ Ori A in the magnetic confinement-rotation diagram (Petit et al. 2013). $\zeta$ Ori A is the only known magnetic massive star with a confinement parameter below 1, i.e. without a magnetosphere. However, Hummel et al. (2013) recently found that $\zeta$ Ori A is a O9.5I+B1IV binary star with a period of $2687.3 \pm 7.0$ days, so the magnetic field of $\zeta$ Ori $\mathrm{A}$ and its confinement parameter might have been wrongly estimated.

\section{Spectropolarimetric analysis}

Spectropolarimetric data were obtained with Narval at TBL between 2008 and 2012. To improve the signal-to-noise ratio, we have coadded spectra obtained on the same night, leading to 36 average spectra. We applied the well-known and commonly used LeastSquares Deconvolution (LSD) technique (Donati et al. 1997) on each average spectrum. The mask is created from a list of lines extracted from VALD (Piskunov et al. 1995; Kupka \& Ryabchikova 1999) using the effective temperature $T_{\text {eff }}=30000 \mathrm{~K}$ and the $\log g=3.25$. We then extracted LSD Stokes I and V profiles for each night as well as null $(\mathrm{N})$ polarization profiles to check for spurious signatures. We see clear Zeeman signatures some nights (see left panel of Fig. 1). $\zeta$ Ori A is thus confirmed to be magnetic. However, $\zeta$ Ori $\mathrm{A}$ is a binary and we ignore which component of the binary is magnetic, or if both

$\dagger$ Postdoctoral Fellow of the Fund for Scientific Research (FWO), Flanders, Belgium 

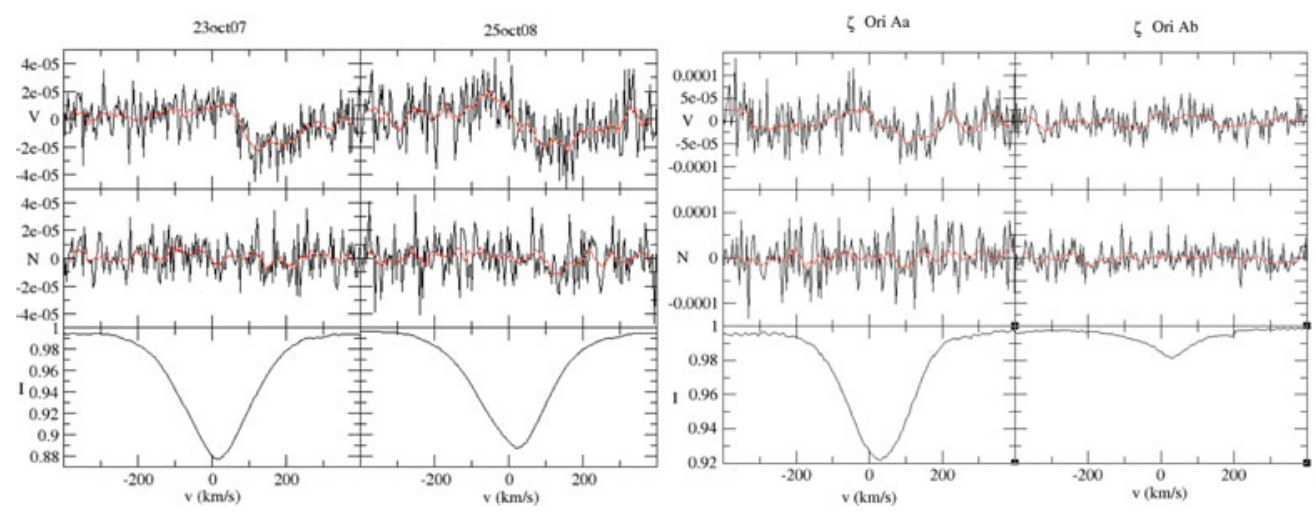

Figure 1. Left: Examples of I (bottom), null N (center) and Stokes V (top) profiles for two nights. Right: I (bottom), null N (center) and Stokes V (top) profiles for October 25, 2008, for $\zeta$ Ori Aa (left) and $\zeta$ Ori Ab (right). The red lines represent the averaged signal.

components are magnetic. To provide an answer to this question we must disentangle the composite spectra.

\section{Disentangling the spectra}

We used the Fourier-based formulation of the spectral disentangling method (Hadrava 1995) as implemented in the FDBINARY code (Ilijic et al. 2004) to try to separate the individual spectra of both components of the $\zeta$ Ori A binary system. This method failed due to a bad phase coverage of the binary period.

Therefore we used a second method to disentangle the spectra: for each component of $\zeta$ Ori A we computed a synthetic spectrum with their respective temperature and log g. Based on these synthetic spectra, we checked which lines in the observations comes from only one of the component. We created two distinct masks with lines from only one of the component and we used LSD with these masks. We find that the magnetic field is present in $\zeta$ Ori Aa and not in $\zeta$ Ori Ab (see right panel of Fig. 1).

\section{Conclusion}

We confirm that $\zeta$ Ori A is a magnetic star. The magnetic field is present in the supergiant $\zeta$ Ori Aa and no magnetic field is detected in its companion $\zeta$ Ori Ab.

\section{References}

Bouret, J.-C., Donati, J.-F., Martins, F., et al. 2008, MNRAS 389, 75

Donati, J.-F., Semel, M., Carter, B. D., Rees, D. E., \& Collier Cameron, A. 1997, MNRAS 291, 658

Hadrava, P. 1995, A\&SAS 114, 393

Hummel, C. A., Rivinius, T., Nieva, M.-F., et al. 2013, A\& $A$ 554, A52

Ilijic, S., Hensberge, H., Pavlovski, K., \& Freyhammer, L. M. 2004, Vol. 318 of ASPCS, p. 111

Kupka, F. \& Ryabchikova, T. A. 1999, Publications de l'Observatoire Astronomique de Beograd 65,223

Petit, V., Owocki, S. P., Wade, G. A., et al. 2013, MNRAS 429, 398

Piskunov, N. E., Kupka, F., Ryabchikova, T. A., Weiss, W. W., \& Jeffery, C. S. 1995, A\&AS 112,525

Wade, G. A., Grunhut, J., Alecian, E., et al. 2013, IAUS 302, ArXiv e-prints 1310.3965 